\title{
Mild Acid Hydrolysis-related Release of Water-soluble Sunscreen Pigments from the Exopolysaccharide Matrix of Edible Terrestrial Cyanobacteria
}

\author{
Wen Liu, Haiyan $\mathrm{Xu}$, Xiang Gao* \\ School of Life Sciences, Central China Normal University, Wuhan 430079, P. R. China.
}

\section{ARTICLE INFO}

Article history:

Received on: 24/06/2017

Accepted on: 11/08/2017

Available online: 30/10/2017

Key words:

Cyanobacteria, Nostoc

flagelliforme, Nostoc commune, sunscreen pigment, acidic

solution, extraction technology.

\begin{abstract}
Terrestrial cyanobacteria, Nostoc flagelliforme and Nostoc commune, are important natural resource for producing ultraviolet-absorbing mycosporine-like amino acids (MAAs), in addition to their edible value. MAAs can be used as sun-screening cosmetics, antioxidants and pharmaceuticals. Usually, water-soluble MAAs are extracted by pure or aqueous methanol after sample grinding. Development of new extraction technique without specific morphological destroyment will contribute to adequately exploit the commercial values of both edible cyanobacteria. The $\mathrm{N}$-acetylcysteine solution has been preliminarily mentioned to cause the release of MAAs from $N$. flagelliforme via mere incubation with the sample. In this study, we explored the potential mechanism underlying this influence and further applied it to extract MAAs from various samples. It was revealed that $\mathrm{N}$-acetylcysteine solutions primarily played a mild acid hydrolysis role in causing the dissociation and release of MAAs from the exopolysaccharide matrix of samples. Nacetylcysteine solutions also exerted similar physiological or morphological effects on the samples as other acidic solutions, and the morphological integrity of the treated samples was not destroyed. Finally, we found that those samples with good vitality could achieve high MAA harvests. In general, means of incubation with acidic solution serves as a simple and relatively nondestructive technique for MAA extraction from the edible terrestrial cyanobacteria.
\end{abstract}

\section{INTRODUCTION}

Mycosporines and mycosporine-like amino acids (MAAs) are small (<400 Da), colorless and water-soluble molecules, with strong ultraviolet (UV) absorption maxima from 310 to $365 \mathrm{~nm}$ $[1,2]$. More than 30 different compounds in the mycosporine family have been identified from natural sources, including cyanobacteria, fungi and marine algae [2,3]. These UV-absorbing molecules (herein all referred to as MAAs) can dissipate the absorbed UV radiation as heat without the generation of reactive oxygen species [4]. They also exhibit antioxidant activity [5,6] and can serve as wound-healing reagents [7] and monoamine oxidase-B inhibitors [8]. Therefore, MAAs have a bright prospect in cosmetic and pharmaceutical industries.

* Corresponding Author

Xiang Gao, School of Life Sciences, Central China Normal University,

Wuhan 430079, P. R. China.Email: ggxx2002 @ 126.com,

Tel: +86-27-67867223
Terrestrial cyanobacteria, Nostoc flagelliforme and Nostoc commune, are important edible resources [9, 10]. The former, which has been overexploited in the past years, is now in restoration in the vast arid or semi-arid steppes of China. The latter is a cosmopolitan species and most abundant in temperate regions. Typically MAAs are free intracellular compounds in marine algae, however they are generally oligosaccharide-linked in the exopolysaccharide (EPS) matrix of terrestrial cyanobacteria [11, $12,13]$. Because of the exposure to intense solar radiation and period desiccation, terrestrial cyanobacteria can be induced to produce rich UV-absorbing MAAs and scytonemin [14, 15]. Thus, edible terrestrial cyanobacteria are also important resources for obtaining MAAs.

The extraction of MAAs from cyanobacteria usually employs pure or aqueous methanol or even water $[1,5,6,13,16$, 17]. Samples are ground and destroyed by these extraction means. Recently, it was preliminarily mentioned that $\mathrm{N}$-acetylcysteine (NAC) solution could cause the release of MAAs from $N$. flagelliforme via only incubation with the sample [12]. It has 
attracted our attention that the relevant technique may be developed for the purpose of deeply exploiting edible terrestrial cyanobacteria without specific morphological destroyment. NAC functions as a precursor of glutathione in cells that can reduce the intracellular oxidant stress and protect the photosystem against UV radiation $[18,19,20]$. NAC can also markedly reduce the intrinsic viscosity of mucoprotein solutions $[21,22]$ and is used as a generic medication to treat acetaminophen overdose or to loosen thick mucus (The American Society of Health-System Pharmacists). However, due to its acidic property, we speculate that NAC solution may exert an acid hydrolysis-related role in the release of cyanobacterial MAAs. In this study, we investigated this potential mechanism and further applied it to extract MAAs in various $N$. flagelliforme and $N$. commune samples with different vitalities.

\section{MATERIALS AND METHODS}

\subsection{Cyanobacterial samples}

$N$. flagelliforme is distributed in arid or semiarid steppes of the west and west-northern parts of China [23]. Four N. flagelliforme samples sourced in 1999, 2004, 2013 and 2016 from Inner Mongolia and one sample sourced in 2016 from Ningxia province were used in this study. $N$. commune is distributed across the whole country. $N$. commune samples sourced at four different regions in 2013, 2014 and 2016 were used in this study. Air-dried samples were immersed in BG1 $1_{0}$ solution overnight (14-20 h) at $22^{\circ} \mathrm{C}$ and $20 \mu \mathrm{mol}$ photons $\mathrm{m}^{-2} \mathrm{~s}^{-1}$ for physiological recovery as described by [24]. After the surface water was absorbed by filter paper, the rewetted samples were subjected to various experiments. Physiological recovery of samples after rehydration was indexed by the Photosystem II (PSII) activity parameter Fv/Fm [24,25], which was detected by a Handy PEA fluorometer according to the manufacturer's specification (Hansatech instruments Ltd., England). The N. flagelliforme sample (Inner Mongolia, 2016) was primarily used for the experiments, which had a well recovered Fv/Fm value of 0.44-0.54.

\subsection{Incubation with NAC or other acidic solutions and MAA detection}

Various solutions used in this study were prepared in BG11 $1_{0}$ solution, including NAC solutions $(0.5-40 \mathrm{mM})$, sulfamic acid solutions (5-40 mM), aspartic acid solutions (5-40 mM) and $50 \mathrm{mM}$ glycine- $\mathrm{HCl}$ buffers $(\mathrm{pH} \mathrm{2.0,} 3.0$ and 4.0). The $\mathrm{pH}$ of each solution was determined by a $\mathrm{pH}$ meter (Mettler Toledo, Germany). The $\mathrm{pH}$ values of $0.5-40 \mathrm{mM}$ NAC solutions range from 3.4 to 2.1. Rewetted samples of $1 \mathrm{~g}$ fresh weight (FW) were incubated with 10 or $20 \mathrm{ml}$ solutions in $50-\mathrm{ml}$ plastic centrifuge tubes. The tubes were placed in an air-conditioned chamber at $22^{\circ} \mathrm{C}$ with occasional shaking (1-2 h interval). After centrifugation at 6,000 rpm (Eppendorf Centrifuge 5810R, Germany) for $5 \mathrm{~min}$, the supernatants were carefully collected and subjected to full- or fixed-wavelength spectroscopic analysis with a UV-2700 Ultraviolet-Visible Spectrophotometer (Shimadzu, Japan). The MAA compounds were characterized by their specific UV- absorption spectrum around $312 \mathrm{~nm}$ in $N$. flagelliforme and $N$. commune $[11,12,26]$.

\subsection{Physiological and morphological analysis}

The physiological inhibitions of acidic solutions on the samples were indexed by the $\mathrm{Fv} / \mathrm{Fm}$ parameter as mentioned above. Briefly, physiologically fully recovered samples were immersed in the solutions for $1 \mathrm{~h}$ and then subjected to $\mathrm{Fv} / \mathrm{Fm}$ detection. The morphological appearance was photographed after samples were incubated with acidic solutions for $3 \mathrm{~h}$. Fresh weight/dry weight (FW/DW) ratio (fold) was calculated by comparing the final weights of rewetted samples (14-20 h immersion) to their original dry weights. For the elasticity analysis, $N$. flagelliforme filaments of $3-5 \mathrm{~cm}$ long were fixed at both ends and stretched until breakage. The stretching rate (fold) was calculated as the ratio of the extended length to the original length.

\section{RESULTS AND DISCUSSION}

\subsection{Mild acid hydrolysis-related release of MAAs}

Various concentrations of NAC solutions were first evaluated in causing the release of MAAs from $N$. flagelliforme (Fig. 1a). A short-time treatment $(1 \mathrm{~h})$ by 10 or $20 \mathrm{mM} \mathrm{NAC}$ solution could obviously lead to the release of MAAs, with an absorbance peak at $312 \mathrm{~nm}$ and a shoulder at around $335 \mathrm{~nm}$ as previously reported $[12,26]$. This characteristic spectrum around $312 \mathrm{~nm}$ represented a group of MAA compounds as indicated by HPLC analysis in $N$. flagelliforme [27]. The case was similar in $N$. commune $[11,13]$. During the extended incubation $(14 \mathrm{~h}), 10$ or 20 mM NAC solution led to more release of MAAs and $5 \mathrm{mM}$ NAC solution was also obviously observed to cause this release. In contrast, normal BG11 $1_{0}$ solution or lower concentrations of NAC solutions $(0.5$ or $1 \mathrm{mM})$ had no such effect after either short-time or extended incubation. NAC solutions are acidic and their acidities are correlated with NAC concentrations. When NAC solutions of different concentrations were buffered to be neutral, they lost this effect (Fig. 1b). Therefore, the role of NAC solution in causing the release of MAAs was closely related to its acidic property. Other acidic solutions were further used to examine this potential role (Fig. 2). Glycine-HCl buffers, sulfamic acid and aspartic acid solutions were used. It was noted that these acidic solutions as well as NAC solutions could be buffered by the EPS matrix to some extent, approaching neutral $\mathrm{pH}$ (e.g., as indicated for glycine-HCl buffers in Fig. 2a). The acid strength-related release of MAAs was still observed for the three groups of acidic solutions. These results implied that acid hydrolysis promoted the dissociation of oligosaccharide-linked MAAs from the EPS matrix. The different molecular effects also existed, to some extent, among these reagents, since the absorbance at $312 \mathrm{~nm}$ of equivalent amounts of MAAs in these acidic solutions varied at a range of $0-20 \%$ (data not shown). In addition, the solution acidity of above pH 5.0 after the EPS buffering seemed not to cause the release of MAAs (Fig. 2). The original pH 3.2 of 1 mM NAC 
solution was buffered to $\mathrm{pH} 5.5$, which solution also had no this effect (in Fig. 1a). Thus, an acidity threshold or enough acid strength was equally critical for the dissociation of MAAs from the EPS matrix. Stronger acids such as hydrofluoric, sulphuric and trifluoroacetic acids are usually adopted to hydrolyze polysaccharides under high temperature for composition analysis $[28,29]$ or hydrolyze the glycosylated MAAs for sugar determination [13]. However, no monosaccharide or oligosaccharide was released in our acid treatment experiments. Therefore, NAC solutions as well as other three acidic solutions in this study exerted a mild acid hydrolysis in causing the dissociation and release of MAAs from the samples.
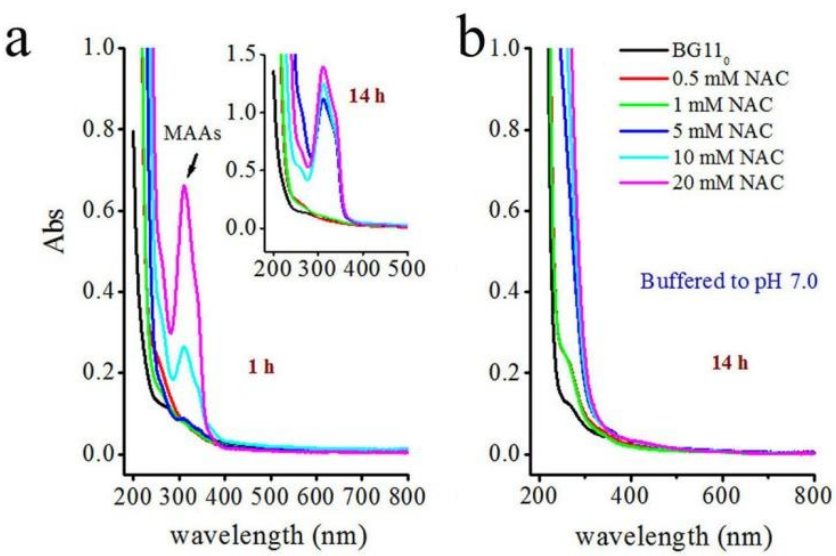

Fig. 1: The spectroscopic scanning of the released MAAs from $N$. flagelliforme samples. a, aliquots of $1 \mathrm{~g} \mathrm{FW}$ rewetted samples were incubated in $10 \mathrm{ml}$ NAC solutions for 1 and $14 \mathrm{~h}$, respectively. b, the rewetted samples were similarly incubated for $14 \mathrm{~h}$ in the NAC solutions ( $\mathrm{pH}$ 7.0) buffered by 20 mM HEPES. The arrow indicates the characteristic absorption peak of MAA compounds at $312 \mathrm{~nm}$.

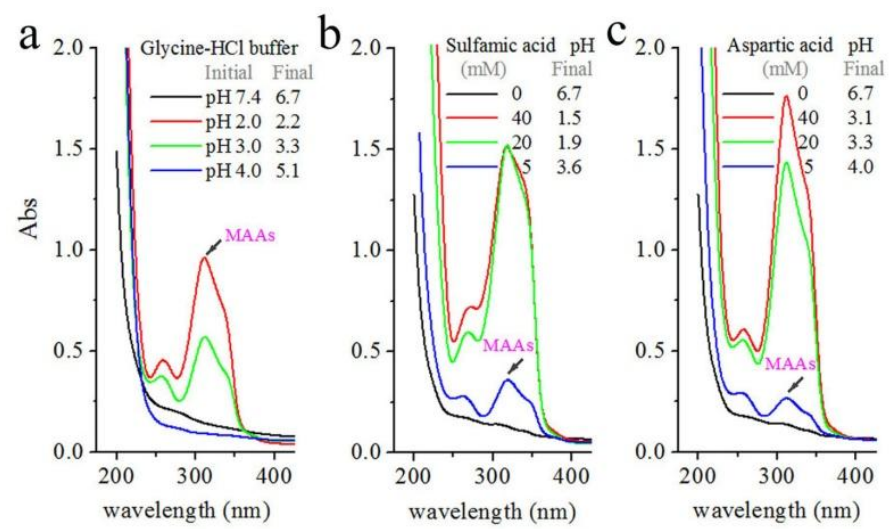

Fig. 2: The release of MAAs from $N$. flagelliforme samples following incubation in various acidic solutions. a, glycine-HCl buffers with $\mathrm{pH} 2.0,3.0$ and 4.0, respectively. b, sulfamic acid solutions of 5, 20 and $40 \mathrm{mM}$, respectively. c, aspartic acid solutions of 5,20 and $40 \mathrm{mM}$, respectively. The rewetted samples of $1 \mathrm{~g} \mathrm{FW}$ were incubated with $10 \mathrm{ml}$ of solutions for $3 \mathrm{~h}$. Due to the $\mathrm{pH}$ buffering of the EPS matrix, the initial and/or final solution $\mathrm{pH}$ was indicated.

\subsection{Physiological or morphological effects of acid treatments}

Physiological or morphological effects of NAC solutions on $N$. flagelliforme samples were evaluated, as compared with glycine- $\mathrm{HCl}$ buffers (Fig. 3). N. flagelliforme and $N$. commune are sensitive to acidic conditions, which lead to physiological inhibition $[23,30]$. As indexed by PSII activity parameter Fv/Fm, NAC solution and glycine- $\mathrm{HCl}$ buffer both resulted in physiological reduction, associated with their concentrations or acidities (Fig. 3a). Moreover, the treated N. flagelliforme filaments by both kinds of solutions showed an increased elasticity (Fig. 3b), implying an obvious softening effect on the EPS matrix. Finally, the morphological appearance of the treated samples seemed not to be particularly affected except the chlorosis at higher acidic conditions (Fig. 3c). The cases were similar for $N$. commune samples (data not shown). These results further demonstrated that NAC solutions exerted a typical acidic effect on cyanobacterial samples. More importantly, acid treatments did not destroy the morphological integrity of the samples. High temperature has a remarkable influence on the stability of MAAs [3]; these compounds will be lost or destroyed in the edible cyanobacteria during the cooking process. Therefore, mere incubation with NAC solution or other acidic solutions contributed to the MAA exploitation from edible cyanobacteria with little influence on their reuse in food business.
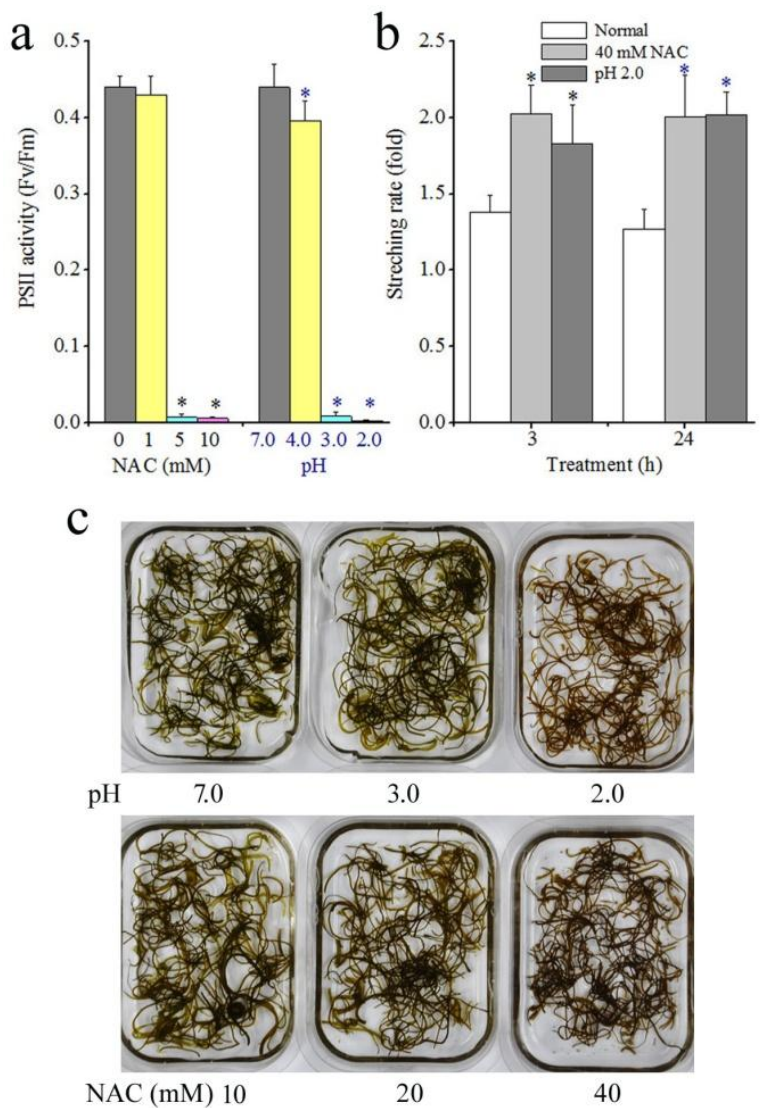

Figure 3 The physiological and morphological effects of acid treatments on $N$. flagelliforme samples. NAC solutions and glycine- $\mathrm{HCl}$ buffers were compared a, PSII activity (in terms of $\mathrm{Fv} / \mathrm{Fm}$ ) changes of the rewetted samples after 1-h incubation in the solutions. Data shown are the means $\pm \mathrm{SD}(\mathrm{n}=5)$. b, elastic changes of the treated $N$. flagelliforme filaments. Control samples are incubated in normal BG11 $1_{0}$ solution. Data shown are the means $\pm \mathrm{SD}(\mathrm{n}=12)$. * indicate significant difference $(\mathrm{P}<0.05)$ with respect to the respective control (student's $t$-test). c, the morphological appearance of the treated samples after incubation for $3 \mathrm{~h}$. 
Table 1: The released MAA contents by NAC solution treatment in various $N$. flagelliforme $(N f)$ and $N$. commune $(N c)$ samples

\begin{tabular}{|c|c|c|c|c|}
\hline $\begin{array}{l}\text { Sample no. } \\
\text { (year, source) }\end{array}$ & $\begin{array}{c}\text { Recovered } \\
\text { Fv/Fm } \\
\end{array}$ & $\begin{array}{c}\text { FW/DW ratio } \\
\text { (fold) }\end{array}$ & $\begin{array}{l}\text { Absorbance } \\
\text { at } 312 \mathrm{~nm}^{\text {b }}\end{array}$ & $\begin{array}{l}\text { MAA content } \\
(\% \text { of DW })^{c}\end{array}$ \\
\hline$N f-1$ 2016, Inner Mongolia & $0.53 \pm 0.04$ & 16.15 & $0.548 \pm 0.011$ & 0.14 \\
\hline Nf-2 2016, Yinchuan, Ningxia & $0.52 \pm 0.02$ & 15.56 & $0.283 \pm 0.019$ & 0.07 \\
\hline Nf-3 2013, Inner Mongolia & $0.10 \pm 0.02$ & 15.96 & $0.086 \pm 0.004$ & 0.02 \\
\hline$N f-4$ 2004, Inner Mongolia & 0 & 13.16 & 0 & l \\
\hline Nf-5 1999, Inner Mongolia & 0 & 15.86 & 0 & l \\
\hline Nc-1 2016, Jingmen, Hubei & $0.44 \pm 0.07$ & 22.15 & $0.921 \pm 0.092$ & 0.31 \\
\hline Nc-2 2014, Wuhan, Hubei & $0.25 \pm 0.08$ & 9.20 & $0.282 \pm 0.008$ & 0.04 \\
\hline Nc-3 2013, Shandan, Gansu & 0 & 20.22 & 0 & / \\
\hline Nc-4 2013, Ninghai, Zhejiang & 0 & 9.46 & 0 & l \\
\hline
\end{tabular}

\subsection{MAA extraction in various samples with different vitalities}

The extraction efficiency of MAAs can be affected by several factors such as reagents, temperature, extraction duration or even sample states (ground or not) $[1,3,6]$. No significant difference in the extraction effect was observed at temperature range of $20-40^{\circ} \mathrm{C}$ or during $2-6 \mathrm{~h}$ incubation (data not shown). Thus, the MAA extraction was still performed at $22^{\circ} \mathrm{C}$ in various $N$. flagelliforme and $N$. commune samples but the incubation was extended to $4 \mathrm{~h}$. To combat the $\mathrm{pH}$ buffering from the EPS matrix, an increased liquid-to-solid ratio was adopted by incubating $1 \mathrm{~g}$ of rewetted samples with $20 \mathrm{ml}$ of $40 \mathrm{mM}$ NAC solution. The released amounts of MAAs in five $N$. flagelliforme and four $N$. commune samples were compared (Table 1). These samples had different vitality (in terms of the recovered extent of $\mathrm{Fv} / \mathrm{Fm}$ ) and moisture absorption capability (FW/DW ratio). Usually, newly collected or well kept $N$. flagelliforme and $N$. commune samples have a good Fv/Fm recovery of above 0.3 . As shown in table 1, the newly collected samples (in 2016) showed overall high MAA contents, ranging from $0.07-0.31 \%$. The big difference in MAA content between two $N$. flagelliforme samples in 2016 possibly implies different environmental influence on its synthesis (e.g., UV intensity and duration of exposure). However, MAAs could not be detected in either $N$. flagelliforme or $N$. commune samples with no photosynthetic physiological recovery. The long-term storage can lead to the reduction or even loss of the vitality of samples [24]. Unlike scytonemin, MAAs are generally instable molecules [3]. Therefore, these results suggested that the timely extraction of MAAs from newly collected samples is beneficial to achieving a relatively high harvest.

\section{CONCLUSION}

In this study, it was revealed that NAC solutions primarily play a mild acid hydrolysis role in causing the dissociation and release of MAAs from the edible terrestrial cyanobacteria. Similar physiological or morphological effects between NAC solutions and other acidic solutions provided further evidence. The $\mathrm{pH}$ buffering role of EPS matrix was also found. However, more importantly, acid treatments via mere incubation did not destroy the morphological integrity of samples, in addition to rapid extraction of MAAs at a temperate condition. Therefore, acid hydrolysis-related MAA extraction serves as a simple and efficient technique for achieving the purpose of deeply exploiting edible terrestrial cyanobacteria. Additionally, it was found that the newly collected samples are an important source for MAA harvest.

\section{ACKNOWLEDGMENTS}

5.1. Financial support and sponsorship: This work has been partially supported by the Fundamental Research Funds for the Central Universities (No. CCNU16A02007).

Conflict of Interests: There are no conflicts of interest.

\section{REFERENCES}

1. Garcia-Pichel F, Castenholz RW. Occurrence of UV-absorbing, mycosporine-like compounds among cyanobacterial isolates and an estimate of their screening capacity. Appl. Environ. Microbiol. 1993; 59: 163-169.

2. Miyamoto KT, Komatsu M, Ikeda H. Discovery of gene cluster for mycosporine-like amino acid biosynthesis from Actinomycetales microorganisms and production of a novel mycosporine-like amino acid by heterologous expression. Appl. Environ. Microbiol. 2014; 80: 5028-5036.

3. Carreto JI, Carignan MO. Mycosporine-like amino acids: relevant secondary metabolites. Chemical and ecological aspects. Mar. Drugs 2011; 9: 387-446.

4. Conde FR, Churio MS, Previtali CM. The photoprotector mechanism of mycosporine-like amino acids. Excited-state properties and photostability of porphyra-334 in aqueous solution. J. Photochem. Photobiol. B. 2000; 56: 139-144.

5. Matsui K, Nazifi E, Kunita S, Wada N, Matsugo S, Sakamoto T. Novel glycosylated mycosporine-like amino acids with radical scavenging activity from the cyanobacterium Nostoc commune. J. Photochem. Photobiol. B. 2001; 105: 81-89.

6. Rastogi RP, Sonani RR, Madamwar D, Incharoensakdi A. Characterization and antioxidant functions of mycosporine-like amino acids in the cyanobacterium Nostoc sp. R76DM. Alg. Res. 2016; 16: 110-118.

7. Choi YH, Yang DJ, Kulkarni A, Moh SH, Kim KW. Mycosporinelike amino acids promote wound healing through focal adhesion kinase (FAK) and mitogen-activated protein kinases (MAP kinases) signaling pathway in keratinocytes. Mar. Drugs 2015; 13: 7055-7066.

8. Scoglio S, Benedetti Y, Benvenuti F, Battistelli S, Canestrari F, Benedetti S. Selective monoamine oxidase B inhibition by an Aphanizomenon flos-aquae extract and by its constitutive active 
principles phycocyanin and mycosporine-like amino acids. Phytomedicine 2014; 21: 992-997.

9. Ninomiya M, Satoh H, Yamaguchi Y, Takenaka H, Koketsu M. Antioxidative activity and chemical constituents of edible terrestrial alga Nostoc commune Vauch. Biosci. Biotechnol. Biochem. 2011; 75: 2175-2177.

10. Gao X, Yang YW, Ai YF, Luo HY, Qiu BS. Quality evaluation of the edible blue-green alga Nostoc flagelliforme using a chlorophyll fluorescence parameter and several biochemical markers. Food Chem. 2014; 143: 307-312.

11. Böhm GA, Pfleiderer W, Böger P, Scherer S. Structure of a novel oligosaccharide-mycosporine-amino acid ultraviolet $\mathrm{A} / \mathrm{B}$ sunscreen pigment from the terrestrial cyanobacterium Nostoc commune. J. Biol. Chem. 1995; 270: 8536-8539.

12. Ferroni L, Klisch M, Pancaldi S, Häder DP. Complementary UVabsorption of mycosporine-like amino acids and scytonemin is responsible for the UV-insensitivity of photosynthesis in Nostoc flagelliforme. Mar. Drugs 2010; 8: 106-121.

13. Nazifi E, Wada N, Asano T, Nishiuchi T, Iwamuro Y, Chinaka S, Matsugo S, Sakamoto T. Characterization of the chemical diversity of glycosylated mycosporine-like amino acids in the terrestrial cyanobacterium Nostoc coтmиe. J. Photochem. Photobiol. B 2015; 142: $154-168$.

14. Wright DJ, Smith SC, Joardar V, Scherer S, Jervis J, Warren A, Helm RF, Potts M. UV irradiation and desiccation modulate the three-dimensional extracellular matrix of Nostoc commune (Cyanobacteria). J. Biol. Chem. 2005; 280: 40271-40281.

15. Fleming ED, Castenholz RW. Effects of periodic desiccation on the synthesis of the UV-screening compound, scytonemin, in cyanobacteria. Environ. Microbiol. 2007; 9: 1448-1455.

16. Volkmann M, Gorbushina AA. A broadly applicable method for extraction and characterization of mycosporines and mycosporinelike amino acids of terrestrial, marine and freshwater origin. FEMS Microbiol. Lett. 2006; 255: 286-295.

17. Rosic NN, Braun C, Kvaskoff D. Extraction and analysis of mycosporine-like amino acids in marine algae. Methods Mol. Biol. 2015; 1308: 119-129.

18. Malanga G, Kozak RG, Puntarulo S. N-acetylcysteine-dependent protection against UV-B damage in two photosynthetic organisms. Plant Sci. 1999; 141: 129-137.

19. Morley N, Curnow A, Salter L, Campbell S, Gould, D. N-Acetyl-Lcysteine prevents DNA damage induced by UVA, UVB and visible radiation in human fibroblasts. J. Photochem. Photobiol. B 2003; 72: $55-60$.
20. Wang G, Hu C, Li D, Zhang D, Li X, Chen K, Liu Y. The response of antioxidant systems in Nostoc sphaeroides against UV-B radiation and the protective effects of exogenous antioxidants. Adv. Space Res. 2007; 39: 1034-1042.

21. Sheffner AL. The reduction in vitro in viscosity of mucoprotein solutions by a new mucolytic agent, N-acetyl-L-cysteine. Ann. N. Y. Acad. Sci. 1963; 106: 298-310.

22. Dasgupta B, King M. Molecular basis for mucolytic therapy. Can. Respir. J. 1995; 2: 223-230.

23. Gao K. Chinese studies on the edible blue-green alga, Nostoc flagelliforme: a review. J. Appl. Phycol. 1998; 10: 37-49.

24. Liu YH, Yu L, Ke WT, Gao X, Qiu BS. Photosynthetic recovery of Nostoc flagelliforme (Cyanophyceae) upon rehydration after 2 years and 8 years dry storage. Phycologia 2010; 49: 429-437.

25. Schuurmans RM, van Alphen P, Schuurmans JM, Matthijs HCP, Hellingwerf KJ. Comparison of the photosynthetic yield of cyanobacteria and green algae: different methods give different answers. PLoS One 2015; 10: e0139061.

26. Feng YN, Zhang ZC, Feng JL, Qiu BS. Effects of UV-B radiation and periodic desiccation on the morphogenesis of the edible terrestrial cyanobacterium Nostoc flagelliforme. Appl. Environ. Microbiol. 2012; 78: 7075-7081.

27. Yu H, Liu R. Effect of UV-B radiation on the synthesis of UVabsorbing compounds in a terrestrial cyanobacterium, Nostoc flagelliforme. J. Appl. Phycol. 2013; 25: 1441-1446.

28. Yu Ip CC, Manam V, Hepler R, Hennessey JP. Carbohydrate composition analysis of bacterial polysaccharides: optimized acid hydrolysis conditions for HPAEC-PAD analysis. Anal. Biochem. 1992; 201: 343-349.

29. Jia S, Yu H, Lin Y, Dai Y. Characterization of extracellular polysaccharides from Nostoc flagelliforme cells in liquid suspension culture. Biotechnol. Bioproc. Eng. 2007; 12: 271-275.

30. Sand-jensen K, Jespersen TS. Tolerance of the widespread cyanobacterium Nostoc commune to extreme temperature variations $\left(-269\right.$ to $\left.105^{\circ} \mathrm{C}\right), \mathrm{pH}$ and salt stress. Oecologia 2012; 169: 331-339.

\section{How to cite this article:}

Liu W, Xu H, Gao X. Mild Acid Hydrolysis-related Release of Water-soluble Sunscreen Pigments from the Exopolysaccharide Matrix of Edible Terrestrial Cyanobacteria. J App Biol Biotech. 2017; 5 (05): 11-15. 